\title{
BMJ Open Hangeshashinto for preventing oral mucositis in patients receiving cancer treatment: protocol for a systematic review and meta-analysis
}

\author{
Yu-Ting Wang (D), Yifeng Ren, Chong Xiao, Hong Liu, Xi Fu, Feng-Ming You
}

To cite: Wang Y-T, Ren Y, Xiao C, et al. Hangeshashinto for preventing oral mucositis in patients receiving cancer treatment: protocol for a systematic review and meta-analysis. BMJ Open 2021;11:e047627. doi:10.1136/ bmjopen-2020-047627

- Prepublication history and additional supplemental material for this paper are available online. To view these files, please visit the journal online (http://dx.doi.org/10.1136/ bmjopen-2020-047627).

YR and F-MY contributed equally.

Received 09 December 2020 Accepted 07 May 2021

\section{ABSTRACT}

Introduction Hangeshashinto has been employed for oral mucositis prevention in patients receiving cancer treatment, but the evidence has not been sufficiently robust to guide clinical decision-making. This study will therefore be undertaken to assess the effectiveness of Hangeshashinto for preventing oral mucositis in patients with cancer who are receiving treatment.

Methods and analysis The databases will include PubMed, Embase, the Cochrane Library, Chinese databases and Japanese databases. The literature will be searched from the databases' inception until May 2021. Other sources, such as potential grey literature, reference lists from included studies and relevant systematic reviews and conference papers, will also be searched. The primary outcome is the incidence of mucositis of any severity, and the secondary outcomes are interruptions to cancer treatment, oral pain and nutritional status. The risk of bias of eligible studies will be assessed using the Cochrane Collaboration's 'risk of bias' tool. Both the $Q$ test and $\mathrm{I}^{2}$ statistic will be performed to assess statistical heterogeneity. If $\left.\right|^{2}>50 \%$, sensitivity and subgroup analysis will be conducted. The quality of evidence will be rated according to the Grading of Recommendations, Assessment, Development and Evaluation approach. Egger's test will be used to assess reporting bias. Ethics and dissemination This systematic review will evaluate only published data; therefore, ethical approval is not required.

PROSPERO registration number CRD42020216145.

\section{INTRODUCTION}

Oral mucositis $(\mathrm{OM})$, a frequent and painful toxicity for patients with cancer, is an inflammatory response of the epithelial mucosa to antineoplastic treatments. Approximately $20 \%-40 \%$ of patients receiving conventional chemotherapy will develop OM, and this percentage increases to $60 \%-85 \%$ in those treated with haematopoietic stem cell transplantation and to $90 \%$ in those with head and neck neoplasms treated with radio-plus chemotherapy. ${ }^{1}$

OM typically manifests as erythema or/ and ulcerations, which may cause pain and

\section{Strengths and limitations of this study}

To the best of our knowledge, this will be the first review to assess the effectiveness of Hangeshashinto as a preventive agent for oral mucositis induced by cancer treatment

- We will retrieve data from only English-language, Japanese-language and Chinese-language databases, which could limit the available data or result in language bias.

- The quality of this study will largely depend on that of the original studies that are included.

difficulty eating, swallowing and talking. ${ }^{2}$ Severe OM increases the requirement for narcotics and enteral nutritional support (including feeding tube use or gastrostomy) and can lead to the interruption of cancer treatments, which compromises tumour control. Furthermore, the ulcer provides an access site for pathogens and may increase the risk of infection or sepsis for immunocompromised patients with cancer. These complications are associated with prolonged hospital stays, increased mortality and increased healthcare costs. ${ }^{34}$ Thus, there has been significant interest in the prevention and treatment of OM.

Despite strategic advances in understanding the molecular basis of OM over the past decade, treatment options are still limited to symptom control with topical agents and systemic pain medication. ${ }^{5}{ }^{6}$ Currently, several interventions and prevention guidelines are available, but their effectiveness is uncertain. It is worth noting that palifermin, the recombinant human keratinocyte growth factor 1 , is the only agent approved by both the US Food and Drug Administration and the European Medical Agency for preventing $\mathrm{OM}$ in patients receiving antineoplastic treatments. ${ }^{6}$ However, due to the high costs and possibility of stimulating cancer cell growth, palifermin is not widely used in the 
clinic for OM management. Other therapies that are more feasible, affordable and accessible to the general public are in urgent demand.

Hangeshashinto (TJ-14), a Kampo medicine containing seven herbal extracts (pinellia tuber, coptis rhizome, scutellaria root, processed ginger, glycyrrhiza, ginseng and jujube), has been approved as a prescribed medicine in Japan and China. ${ }^{78}$

Since the 16th century, TJ-14 has been widely used to improve inflammation-associated gastrointestinal symptoms, such as refractory gastro-oesophageal reflux disease, diarrhoea and $\mathrm{OM}^{8-10}$ Several clinical studies have reported that repetitive mouth washing with TJ-14 significantly reduces $\mathrm{OM}$ scores and improves cancer treatment completion rates. ${ }^{11-13}$ Recently, in vitro and in vivo experiments have revealed the underlying mechanisms by which TJ-14 improves OM, which is related to anti-inflammatory and antibacterial effect. ${ }^{14-16}$ The results of these studies suggest that TJ-14 may provide a cheap and effective alternative for preventing or minimising $\mathrm{OM}$, but no systematic review so far has authenticated the correlation between TJ-14 use and OM. Therefore, the purpose of this systematic review is to provide a retrospective investigation of the current evidence regarding the effectiveness of the application of TJ-14 for preventing OM in patients receiving cancer treatments to guide clinical decisions.

\section{Objectives}

To assess the effectiveness of TJ-14 in preventing OM in patients who are receiving treatment for cancer.

\section{METHODS AND ANALYSIS}

This study will be performed in agreement with Preferred Reporting Items for Systematic Review and Meta-Analysis Protocols (PRISMA-P) guidelines ${ }^{17}$ using the Population, Intervention, Comparison, Outcomes and Study framework. ${ }^{18}$ The PRISMA-P checklist and the PRISMA flow chart for the primary literature selection process are shown in online supplemental checklist and figure.

\section{Search strategy}

A systematic electronic search will be conducted in the following databases: PubMed, Embase, Cochrane Library, Chinese databases (Chinese National Knowledge Infrastructure (CNKI) and Wan-Fang Database) and Japanese databases (Citation Information by Nii (CiNii) and Ichushi-web). The reference lists from eligible studies and relevant systematic reviews will be searched manually, and relevant conference proceedings will also be searched. Additionally, we plan to search OpenGrey.eu for potential grey literature to ensure completeness of the literature search.

The retrieval time will be set from the time of database inception to May 2021. The publication languages will be limited to English, Japanese or Chinese. The following terms will be used for the search: neoplasms, cancer, tumor, leukemia, lymphoma, radiotherapy, antineoplastic agents, oral mucositis, stomatitis, Hangeshashinto and TJ-14. The PubMed search strategy is

\begin{tabular}{|c|c|}
\hline Number & Search terms \\
\hline$\# 1$ & $\begin{array}{l}\text { (Neoplasms[mesh]) OR (Neoplas*[ti/ab]) OR } \\
\text { (Tumor*[ti/ab]) OR (Tumour*[ti/ab]) OR (cancer*[ti/ } \\
\text { ab]) OR (Malignanc*[ti/ab]) OR (Malignant*[ti/ab]) }\end{array}$ \\
\hline$\# 2$ & $\begin{array}{l}\text { (Leukemia[mesh]) OR (Leukemias[ti/ab]) OR } \\
\text { (Leucocythaemia[ti/ab]) OR (Leucocythemia[ti/ } \\
\text { ab]) }\end{array}$ \\
\hline$\# 3$ & $\begin{array}{l}\text { (Lymphoma[mesh]) OR (Lymphoma*[ti/ } \\
\text { ab]) OR (Germinoblast*[ti/ab]) OR } \\
\text { (Reticulolymphosarcoma*[ti/ab]) }\end{array}$ \\
\hline$\# 4$ & $\begin{array}{l}\text { (Radiotherapy[mesh]) OR (Radiotherap*[ti/ab]) } \\
\left.\text { OR ((Therap*[ti/ab]) OR (Treatment* }{ }^{*}[\mathrm{t} / \mathrm{ab}]\right) \text { AND } \\
\left.\left.\text { (Radiation* }{ }^{*}[\mathrm{ti} / \mathrm{ab}]\right)\right)\end{array}$ \\
\hline$\# 5$ & $\begin{array}{l}\text { (Antineoplastic Agents[mesh]) OR } \\
\text { (Antineoplastic*[ti/ab]) OR (((Anticancer[ti/ab]) } \\
\text { OR (Antitumor[ti/ab]) OR (Cancer[ti/ab])) AND } \\
((\text { Agent*[ti/ab]) OR (Drug*[ti/ab]))) }\end{array}$ \\
\hline$\# 6$ & $\begin{array}{l}\text { (Bone Marrow Transplantation[mesh]) OR } \\
((\text { Bone Marrow[ti/ab]) AND ((Grafting[ti/ab]) OR } \\
\text { (Transplantation[ti/ab]))) }\end{array}$ \\
\hline$\# 7$ & $\begin{array}{l}\text { (Stomatitis[mesh]) OR (Stomatitides[ti/ab]) } \\
\text { OR (Oral Mucositis[ti/ab]) OR (Mucositides, } \\
\text { Oral[ti/ab]) OR (Oral Mucositides[ti/ab]) OR } \\
\text { (Oromucositis[ti/ab]) }\end{array}$ \\
\hline$\# 8$ & $\begin{array}{l}\text { (Hangeshashinto[ti/ab]) OR (TJ-14[ti/ab]) OR } \\
\text { (Banxia Xiexin[ti/ab]) OR (Banxiaxiexin*[ti/ab]) OR } \\
\text { (Ban Xia Xie Xin[ti/ab]) OR (Banha-Sasim-Tang[ti/ } \\
\text { ab]) OR (Pinellia Heart-draining[ti/ab]) OR (Pinellia } \\
\text { Decoction for Draining the Heart[ti/ab]) }\end{array}$ \\
\hline$\# 9$ & $\begin{array}{l}\text { (\#1 OR \#2 OR \#3 OR \#4 OR \#5 OR \#6 OR \#7) } \\
\text { AND \#8 }\end{array}$ \\
\hline
\end{tabular}

shown in table 1. The search strategies used for Embase, Cochrane Library, CNKI, Wan-Fang Database, CiNii and Ichushi-web are supplied in online supplemental search strategies.

\section{Eligible criteria}

Type of studies

All randomised controlled trials (RCTs) and quasi-RCTs published since the time of database inception to May 2021 will be included.

\section{Type of participants}

Patients receiving cancer treatments will be included.

\section{Type of interventions and comparisons}

All studies comparing TJ-14 with placebo, no treatment, usual care or any other approach to prevent OM induced by cancer treatments will be included in this study.

We will exclude studies of combination interventions for the prevention of OM because it may be difficult to attribute any effect expressed to a specific component of the intervention. 
Types of outcome measures

\section{Primary outcomes}

The primary outcome is the incidence of OM of any severity. We are interested in both the presence/absence of OM, and also different levels of severity. Two of the most commonly used scales for OM are the WHO classification $^{19}$ and National Cancer Institute Common Terminology Criteria for Adverse Events. ${ }^{20} 21$ It will be measured on a scale of 0-4 (none to severe) and be dichotomised as no mucositis versus any mucositis ( 0 vs $1+)$, mild versus moderate to severe mucositis ( $0-1$ vs $2+)$, and mild to moderate versus severe mucositis $(0-2$ vs $3+)$.

\section{Secondary outcomes}

1. Interruptions to cancer treatment.

2. Oral pain: the Numeric Rating Scale ${ }^{22}$ and the Visual Analogue Scale ${ }^{19}$ will be used to evaluate oral pain.

3. Nutritional status: including bodyweight reduction, serum albumin levels, feeding tube or gastrostomy use.

\section{Selection studies}

Two review authors (Y-TW and CX) will independently screen the titles and abstracts of studies retrieved from the electronic searches. We will obtain the full text of all studies that appear to meet the inclusion criteria for further review and assessment. Any disputes will be resolved through discussion or consultation with the third review author (YR) if we are unable to resolve disagreements. Excluded studies will be recorded along with the reasons for exclusion.

\section{Data extraction and coding}

Two review authors (Y-TW and CX) will independently extract data from all included studies using a data extraction form, which will include the following information: the first author, the year of publication, study population, study design, the number of total and analysed patients, baseline analysis, blinding method, imputation method, interventions, controls, and primary and secondary outcomes. To investigate the characteristics of the TJ-14 effect, we will also extract data on sex, age, features of cancer (such as primary site, cancer stage, cancer type and duration from the first diagnosis), features of cancer treatments (such as form, dose and frequency), and control intervention details. We will contact the study authors for missing data. Any disagreement will be resolved through discussion or consultation with the third review author (YR) to achieve consensus if necessary.

\section{Quality assessment}

Two separate review authors (HL and XF) will independently assess the risk of bias for eligible studies using the Cochrane Collaboration's risk of bias tool. ${ }^{23}$ Six major domains will be assessed for each trial, including random sequence generation, allocation concealment, blinding method, incomplete outcome data, selective reporting and other sources of bias. All studies will be categorised into three grades (low, high and unclear risk of bias). Any disagreement will be resolved through discussion and consultation with the third review author (F-MY) if necessary. RevMan V.5.3.5 will be used to generate the graphical presentation of the assessment of risk of bias.

\section{Statistical analysis}

To determine the distinctions between the intervention and control groups, $\mathrm{OM}$ incidence of any severity at the completion of cancer treatments and at the end of follow-up will be extracted as a primary outcome. The number of interruptions to cancer treatment, incidence of oral pain, nutrition status, adverse events, number of hospitalisation days and number of days intervention with analgesics will be included as secondary outcomes. The incidence of $\mathrm{OM}$ will be measured on a scale of $0-4$ (none to severe) and be dichotomised as no mucositis versus any mucositis ( 0 vs $1+$ ), mild versus moderate to severe mucositis (0-1 vs $2+$ ), and mild to moderate versus severe mucositis (0-2 vs $3+)$. For dichotomous outcomes (eg, mucositis of any severity vs none), the Relative Risk (RR) with $95 \%$ CI will be used to express the effect of TJ-14 treatment. For continuous outcomes (eg, number of hospitalisation days), the Mean Deviation (MD) with $95 \%$ CI will be extracted and calculated as an effect estimate.

Both the $Q$ test and $\mathrm{I}^{2}$ statistic will be performed for the assessment of statistical heterogeneity. Values of $\mathrm{I}^{2}$ equal to or greater than $50 \%$ will be considered to represent substantial heterogeneity, and values equal to or greater than $75 \%$ indicate considerable heterogeneity. ${ }^{24}$ We will explore the sources of heterogeneity by conducting subgroup analysis and sensitivity analysis. Funnel plots will be used for the assessment of reporting biases and Egger's test will be performed to evaluate funnel plot asymmetry. The meta-analysis results will be displayed in a forest plot with $95 \%$ CIs. The Grading of Recommendations, Assessment, Development and Evaluation approach ${ }^{25}$ will be used to summarise the quality of the evidence for the obtained results. We will categorise the overall quality of evidence as high, moderate, low or very low.

\section{Patient and public involvement}

No patient and public involved.

\section{DISCUSSION}

$\mathrm{OM}$ is a significant complication of cancer therapy, affecting more than $75 \%$ of high-risk patients. ${ }^{2}$ TJ-14 has been widely used for prevention and treatment of OM, but the evidence has not been sufficiently robust to guide clinical decision-making. This systematic review and meta-analysis will provide an assessment of the current state of TJ-14 for OM. Conclusions drawn from this study may benefit patients with OM, clinicians and policymakers. The process of this review will comprise four sections: identification, study inclusion, data extraction and data synthesis. Two main potential limitations may affect the conclusions. First, we will retrieve data from 
only English-language, Japanese-language and Chineselanguage databases, which could limit the available data or result in language bias. Second, the quality of original studies may be poor, limiting the ability to generate conclusions based on high confidence.

\section{ETHICS AND DISSEMINATION}

Ethical approval is not required since this review will only include published data which already received ethical approval prior to publication.

Acknowledgements This work was supported by the China Academy of Chinese Medical Sciences, China Center for Evidence Based Traditional Chinese Medicine.

Contributors Conception and design-F-MY and YR. Administrative support-FMY. Provision of study materials or patients-Y-TW, CX and YR. Collection and assembly of data-Y-TW, CX and YR. Data analysis and interpretation- HL, XF and F-MY. Manuscript writing —all authors. Final approval of manuscript—all authors.

Funding This work was supported by the National Administration of Traditional Chinese Medicine (TCM): 2019 project of building evidence based practice capacity for TCM (grant number: 2019XZZX-ZL006); National Natural Science Foundation of China (grant number: 81774284 ); Project of Science and Technology Department of Sichuan Province (grant number: 2020098); and Provincial Developmental Fund of TCM-Key Discipline of TCM (Oncology of TCM) (grant number: 2100601).

Competing interests None declared.

Patient consent for publication Not required.

Provenance and peer review Not commissioned; externally peer reviewed.

Supplemental material This content has been supplied by the author(s). It has not been vetted by BMJ Publishing Group Limited (BMJ) and may not have been peer-reviewed. Any opinions or recommendations discussed are solely those of the author(s) and are not endorsed by BMJ. BMJ disclaims all liability and responsibility arising from any reliance placed on the content. Where the content includes any translated material, BMJ does not warrant the accuracy and reliability of the translations (including but not limited to local regulations, clinical guidelines, terminology, drug names and drug dosages), and is not responsible for any error and/or omissions arising from translation and adaptation or otherwise.

Open access This is an open access article distributed in accordance with the Creative Commons Attribution Non Commercial (CC BY-NC 4.0) license, which permits others to distribute, remix, adapt, build upon this work non-commercially, and license their derivative works on different terms, provided the original work is properly cited, appropriate credit is given, any changes made indicated, and the use is non-commercial. See: http://creativecommons.org/licenses/by-nc/4.0/.

ORCID iD

Yu-Ting Wang http://orcid.org/0000-0002-7267-4026

\section{REFERENCES}

1 Villa A, Sonis ST. Pharmacotherapy for the management of cancer regimen-related oral mucositis. Expert Opin Pharmacother 2016;17:1801-7.

2 Scully C, Sonis S, Diz PD. Oral mucositis. Oral Dis 2006;12:229-41.

3 Murphy BA, Beaumont JL, Isitt J, et al. Mucositis-related morbidity and resource utilization in head and neck cancer patients receiving radiation therapy with or without chemotherapy. J Pain Symptom Manage 2009;38:522-32.

4 Sonis ST, Elting LS, Keefe D, et al. Perspectives on cancer therapyinduced mucosal injury: pathogenesis, measurement, epidemiology, and consequences for patients. Cancer 2004;100:1995-2025.

5 Elad S, Cheng KKF, Lalla RV, et al. MASCC/ISOO clinical practice guidelines for the management of mucositis secondary to cancer therapy. Cancer 2020;126:4423-31.
6 Peterson DE, Boers-Doets CB, Bensadoun RJ, et al. Management of oral and gastrointestinal mucosal injury: ESMO clinical practice guidelines for diagnosis, treatment, and follow-up. Ann Oncol 2015;26:v139-51.

7 Ozawa N, Onda T, Hayashi K, et al. Effects of topical Hangeshashinto (TJ-14) on chemotherapy-induced oral mucositis. Cancer Manag Res 2020;12:1069-78.

8 Ohnishi S, Takeda H. Herbal medicines for the treatment of cancer chemotherapy-induced side effects. Front Pharmacol 2015;6:14

9 Takeuchi T, Hongo H, Kimura T, et al. Efficacy and safety of hangeshashinto for treatment of GERD refractory to proton pump inhibitors : Usual dose proton pump inhibitors plus hangeshashinto versus double-dose proton pump inhibitors: randomized, multicenter open label exploratory study. $J$ Gastroenterol 2019:54:972-83.

10 Miyashita T, Kono T, Matsui D, et al. Preventive effect of oral hangeshashinto (TJ-14) on the development of reflux-induced esophageal cancer. Surgery 2018:55 doi:10.1016/j.surg.2018.02.003

11 Nishikawa K, Aoyama T, Oba MS, et al. The clinical impact of Hangeshashinto (TJ-14) in the treatment of chemotherapyinduced oral mucositis in gastric cancer and colorectal cancer: analyses of pooled data from two phase II randomized clinical trials (HANGESHA-G and HANGESHA-C). J Cancer 2018;9:1725-30.

12 Yamashita T, Araki K, Tomifuji M, et al. A traditional Japanese medicine-Hangeshashinto (TJ-14)-alleviates chemoradiation-induced mucositis and improves rates of treatment completion. Support Care Cancer 2015;23:29-35.

13 Matsuda C, Munemoto Y, Mishima H, et al. Double-blind, placebocontrolled, randomized phase II study of TJ-14 (Hangeshashinto) for infusional fluorinated-pyrimidine-based colorectal cancer chemotherapy-induced oral mucositis. Cancer Chemother Pharmacol 2015;76:97-103.

14 Miyano K, Eto M, Hitomi S, et al. The Japanese herbal medicine Hangeshashinto enhances oral keratinocyte migration to facilitate healing of chemotherapy-induced oral ulcerative mucositis. Sci Rep 2020;10:625.

15 Hitomi S, Ono K, Terawaki K, et al. [6]-gingerol and [6]-shogaol, active ingredients of the traditional Japanese medicine hangeshashinto, relief oral ulcerative mucositis-induced pain via action on $\mathrm{Na}^{+}$channels. Pharmacol Res 2017;117:288-302.

16 Kono T, Kaneko A, Matsumoto C, et al. Multitargeted effects of hangeshashinto for treatment of chemotherapy-induced oral mucositis on inducible prostaglandin E2 production in human oral keratinocytes. Integr Cancer Ther 2014;13:435-45.

17 Shamseer L, Moher D, Clarke M, et al. Preferred reporting items for systematic review and meta-analysis protocols (PRISMA-P) 2015 elaboration and explanation. BMJ 2015;349:97647.

18 Moher D, Liberati A, Tetzlaff J, et al. Preferred reporting items for systematic reviews and meta-analyses: the PRISMA statement. PLoS Med 2009;6:e1000097.

19 Oton-Leite AF, Corrêa de Castro AC, Morais MO, et al. Effect of intraoral low-level laser therapy on quality of life of patients with head and neck cancer undergoing radiotherapy. Head Neck 2012;34:398-404.

20 Brizel DM, Murphy BA, Rosenthal DI, et al. Phase II study of palifermin and concurrent chemoradiation in head and neck squamous cell carcinoma. J Clin Oncol 2008;26:2489-96.

21 Dazzi C, Cariello A, Giovanis P, et al. Prophylaxis with GM-CSF mouthwashes does not reduce frequency and duration of severe oral mucositis in patients with solid tumors undergoing highdose chemotherapy with autologous peripheral blood stem cell transplantation rescue: a double blind, randomized, placebocontrolled study. Ann Oncol 2003;14:559-63.

22 Arora H, Pai KM, Maiya A, et al. Efficacy of He-Ne laser in the prevention and treatment of radiotherapy-induced oral mucositis in oral cancer patients. Oral Surg Oral Med Oral Pathol Oral Radiol Endod 2008;105:180-6.

23 Hugues A, Di Marco J, Janiaud P, et al. Efficiency of physical therapy on postural imbalance after stroke: study protocol for a systematic review and meta-analysis. BMJ Open 2017;7:e013348.

24 Higgins JPT, Thompson SG, Deeks JJ, et al. Measuring inconsistency in meta-analyses. BMJ 2003;327:557-60.

25 Guyatt GH, Oxman AD, Schünemann HJ, et al. GRADE guidelines: a new series of articles in the Journal of clinical epidemiology. J Clin Epidemiol 2011;64:380-2. 